archives

of thermodynamics

Vol. 38(2017), No. 3, 119-134

DOI: $10.1515 /$ aoter-2017-0019

\title{
Analysis of heat flow in a tube bank of a condenser considering the influence of air
}

\author{
MAGDA JOACHIMIAK* \\ DAMIAN JOACHIMIAK \\ PIOTR KRZYŚLAK
}

Poznan University of Technology, Chair of Thermal Engineering, Piotrowo 3, 60-965 Poznań, Poland

\begin{abstract}
The pressure of wet water vapor inside a condenser has a great impact on the efficiency of thermal cycle. The value of this pressure depends on the mass share of inert gases (air). The knowledge of the spots where the air accumulates allows its effective extraction from the condenser, thus improving the conditions of condensation. The condensation of water vapor with the share of inert gas in a model tube bank of a condenser has been analyzed in this paper. The models include a static pressure loss of the water vapor/air mixture and the resultant changes in the water vapor parameters. The mass share of air in water vapor was calculated using the Dalton's law. The model includes changes of flow and thermodynamic parameters based on the partial pressure of water vapor utilizing programmed water vapor tables. In the description of the conditions of condensation the Nusselts theory was applied. The model allows for a deterioration of the heat flow conditions resulting from the presence of air. The paper contains calculations of the water vapor flow with the initial mass share of air in the range 0.2 to $1 \%$. The results of calculations clearly show a great impact of the share of air on the flow conditions and the deterioration of the conditions of condensation. The data obtained through the model for a given air/water vapor mixture velocity upstream of the tube bank allow for identification of the spots where the air accumulates.
\end{abstract}

Keywords: Condensation; Inert gas; Mass share of gas noncondensing in water vapor; Dalton's law; Water vapor and air partial pressure

${ }^{*}$ Corresponding Author. Email: magda.joachimiak@put.poznan.pl 


\section{Nomenclature}

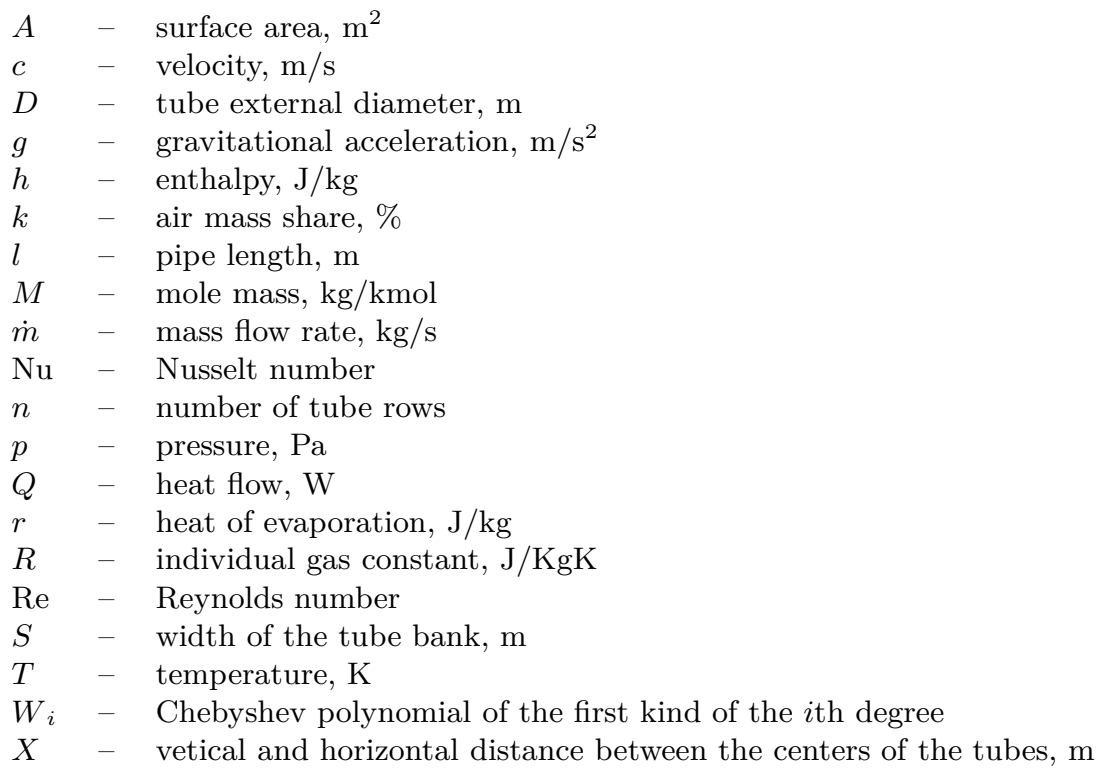

\section{Greek symbols}

$\alpha \quad-$ heat transfer coefficient, $\mathrm{W} / \mathrm{m}^{2} \mathrm{~K}$

$\Delta-$ difference

$\varepsilon \quad-\quad$ function describing the relation of heat transfer coefficients for condensation of water vapor with air and with pure water vapor

$\lambda-$ heat conductance coefficient, $\mathrm{W} / \mathrm{mK}$

$\mu \quad-$ dynamic viscosity, $\mathrm{kg} / \mathrm{m} \mathrm{s}$

$\nu \quad-\quad$ kinematic viscosity, $\mathrm{m}^{2} / \mathrm{s}$

$\rho \quad-$ density, $\mathrm{kg} / \mathrm{m}^{3}$

$\xi \quad-\quad$ total coefficient of pressure loss

\section{Superscripts}

$0 \quad-\quad$ upstream of the first row of tubes

a $\quad-$ air

$c \quad-\quad$ condensed

$f \quad-$ flow

$l \quad-\quad$ condensate

$m \quad-\quad$ mixture of gases

$s \quad-$ saturation

$p \quad-\quad$ tubes in one bank row

sum - total for all rows of tubes

$v \quad-$ water vapor

$w \quad-\quad$ wall 


\section{Introduction}

The efficiency of thermal cycle depends, among others things, on the vacuum level inside the condenser. Due to the leakage of the condenser, air (inert gas) penetrates into the system. One of the factors influencing pressure and the resulting temperature of water vapor saturation is the mass share of inert gases $[4,6,8]$. The presence of air results in a decrease of the partial pressure of water vapor near the condensate film, which causes a local drop of saturation temperature, decreases the difference of temperatures between the water vapor and the wall and deteriorates the conditions of heat flow $[5,6,8,10]$ (Fig. 1).

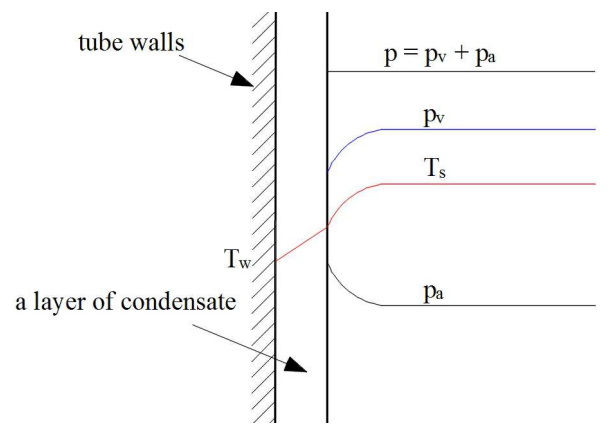

Figure 1: Distribution of pressure and temperature during condensation of water vapor in a solution with air [10].

The accumulating air causes additional thermal resistance and necessitates a greater number of tube rows for the water vapor mass flow to condense. In [3] heat flow and the phenomenon of subcooling was analyzed for condensation inside the horizontal tube. The influence of the share of air, gas velocity and pressure on the heat transfer coefficient was investigated. The influence of the mass flow of gas, mass share of inert gas and pressure during condensation inside the horizontal tube on the heat flow was described in [1]. Experimental research indicates that an increase in the mass share of air to $4 \%$ results in a drop of the heat transfer coefficient, $\alpha$, of up to $20 \%$ referred to the same coefficient for pure water vapor [2]. Problems related to the mathematical modeling of condensers have been described in [8]. In this work a mathematical model was analyzed that leads to a solution in the form of fields of velocity, pressure and inert gases for two types of condensers. The analysis of the changes of thermodynamic parameters during water vapor condensation with the participation of air is an important issue 
in terms of water vapor cycle efficiency. In this paper the authors analyze the influence of the mass share of air in water vapor on the thermal and flow parameters in the model tube bank of a condenser.

\section{Calculation model}

The model tube bank is composed of tubes set in a rectangular order with identical vertical and horizontal distance (Fig. 2). The calculations were made for $l=1 \mathrm{~m}$ of the tube length and the width of the tube bank $S$. Hence, the flow surface area upstream of the bank is

$$
A_{0}=S l .
$$

The flow surface area of the gas among the tubes can be described with the formula

$$
A_{f}=\frac{S(X-D) l}{X} .
$$

The lateral area of the tubes in one row is

$$
A_{p}=\frac{S}{X} \pi D l .
$$

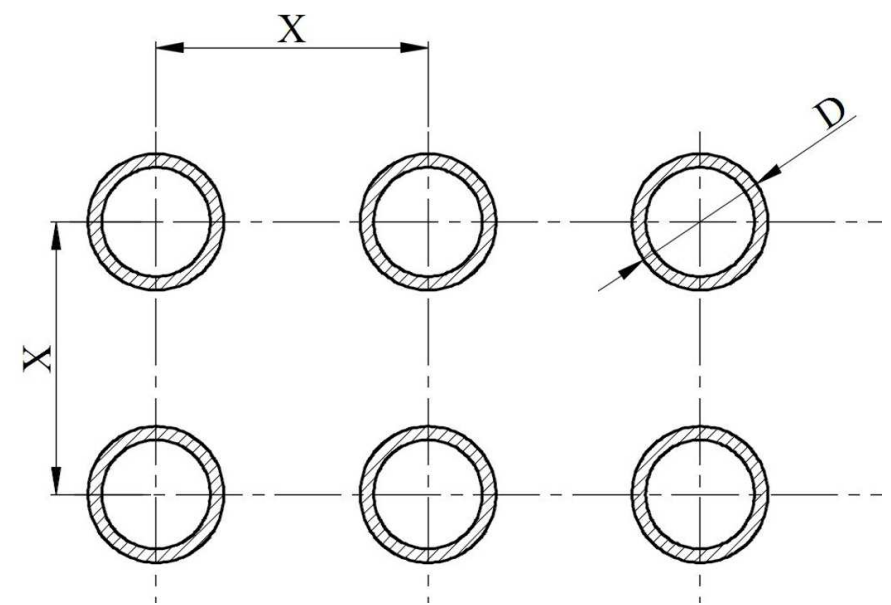

Figure 2: Distribution of the tubes in the tube bank.

The following values have been assumed upstream of the first row of tubes: gas mixture pressure, velocity of gases and mass share of air and temperature of the tube walls. In the calculations, water vapor tables have been 
used based on $[9,11]$. Based on the partial pressure of water vapor in an $i$ th row of tubes, for the saturation parameters, saturation temperature, $T_{v i}$, specific enthalpy, $h_{1 i}$, specific volume, $v_{v i}$, heat of evaporation, $r_{i}$, and kinematic viscosity, $\nu_{v i}$, were calculated. For the given partial pressure of water vapor from the saturation line $x=0$ specific volume $v_{l i}$ and kinematic viscosity, $\mu_{l i}$, of the condensate were obtained. In the model, the authors assumed that the temperature of the mixture equals that of the water vapor saturation

$$
T_{m i}=T_{v i} .
$$

If the calculated value of the temperature of water vapor saturation in an $i$ th row of tubes is lower or equal to the temperature of the tube walls it will result in stopping of the calculations. The mass flow of the gases in an $i$ th row of tubes was determined as a difference of the mass flow of gases in the previous row less the condensed mass flow of water vapor

$$
\dot{m}_{i}=\dot{m}_{i-1}-\Delta \dot{m}_{i-1} .
$$

For the first row of tubes the mass flow rate was determined with the formula

$$
\dot{m}_{1}=\rho_{m 1} c_{1} A
$$

and for the last row

$$
\dot{m}_{m}=k_{a 1} \dot{m}_{1} .
$$

The share of air in the $i$ th row of tubes is

$$
k_{a i}=\frac{k_{a 1} \dot{m}_{1}}{\dot{m}_{i}} .
$$

For the first row of tubes the share of air $k_{a 1}$ is a preset value while for the last row $k_{a m}=1$ (for rows in which the entire mass flow rate of steam is condensed). Hence, the mass airflow in the $i$ th row of tubes is

$$
\dot{m}_{a i}=k_{a i} \dot{m}_{i} .
$$

The gas constant of the mixture of gases depends on gas constants of air and water vapor as well as the mass share of air in the water vapor

$$
R_{m i}=R_{a} k_{a i}+R_{v}\left(1-k_{a i}\right),
$$

where $R_{a}=278.05 \frac{\mathrm{J}}{\mathrm{kgK}}$ while $R_{v}=461.91 \frac{\mathrm{J}}{\mathrm{kgK}}$. The pressure of the mixture of gases in the $i$ th row of tubes was determined based on the difference 
between the pressure of gases upstream of the $i$ th row of tubes and the drop of pressure in this row

$$
p_{m i}=p_{m, i-1}-\Delta p_{m, i-1} .
$$

For the first row of tubes the mixture pressure, $p_{m 1}$, is a preset value. The partial pressure of air was determined based on the Clapeyron equation

$$
p_{a i}=\frac{k_{a i} p_{m i} R_{a}}{R_{v}+k_{a i}\left(R_{a}-R_{v}\right)}=\frac{k_{a i} p_{m i} R_{a}}{R_{m i}} .
$$

Based on the Dalton's law, the partial pressure of water vapor was calculated

$$
p_{v i}=p_{m i}-p_{a i} .
$$

The density of air is

$$
\rho_{a i}=\frac{p_{a i}}{R_{a} T_{m i}} .
$$

From the Dalton's law and the Clapeyron equation, the density of mixture of gases was determined

$$
\rho_{m i}=\frac{R_{a} \rho_{a i}+R_{v} \rho_{v i}}{R_{m i}} .
$$

The dynamic viscosity was determined based on the steam tables, and the air viscosity was based on the air density (14) as well as the relation describing kinematic viscosity of air. The air viscosity is described by the function depending on temperature of the gas mixture

$$
\nu_{a i}=0.0016583 e^{-0.053\left[T_{m}(i-1)-273.15\right]} .
$$

The dynamic viscosity has been described with the Wilkie method [10]

$$
\mu_{m i}=\frac{z_{v i} \mu_{v i}}{z_{v i} \Phi_{v v i}+z_{a i} \Phi_{v a i}}+\frac{z_{a i} \mu_{a i}}{z_{v i} \Phi_{a v i}+z_{a i} \Phi_{a a i}},
$$

where

$$
\begin{gathered}
\Phi_{v v i}=\Phi_{a a i}=1, \\
\Phi_{v a i}=\frac{1}{\sqrt{8}}\left(1+\frac{M_{v}}{M_{a}}\right)^{-0.5}\left[1+\left(\frac{\mu_{v i}}{\mu_{a i}}\right)^{0.5}\left(\frac{M_{a}}{M_{v}}\right)^{0.25}\right]^{2}, \\
\Phi_{a v i}=\frac{1}{\sqrt{8}}\left(1+\frac{M_{a}}{M_{v}}\right)^{-0.5}\left[1+\left(\frac{\mu_{a i}}{\mu_{v i}}\right)^{0.5}\left(\frac{M_{v}}{M_{a}}\right)^{0.25}\right]^{2},
\end{gathered}
$$




$$
z_{v i}=\frac{p_{v i}}{p_{m i}}, \quad z_{a i}=\frac{p_{a i}}{p_{m i}} .
$$

Viscosity of the mixture is determined by the relation [10]

$$
\nu_{m i}=\frac{\mu_{m i}}{\rho_{m i}} .
$$

Based on the flow continuity equation, the velocities of gases among the tubes can be expressed as

$$
c_{i}=\frac{\dot{m}_{i}}{\rho_{m i} A_{f}} .
$$

The total coefficient of pressure loss in the $i$ th row of tubes was described with the relation [2]

$$
\xi_{\text {sum }, i}=(6+9 i)\left(\frac{X}{D}\right)^{-0.13} \operatorname{Re}_{i}^{-0.26},
$$

where $\operatorname{Re}_{i}=\frac{D c_{i}}{\nu_{m i}}$. The loss of pressure is

$$
\Delta p_{m i}=\left(\xi_{i}-\xi_{i-1}\right) \frac{\rho_{m i} c_{i}^{2}}{2}
$$

where $\xi_{0}=0$. The Nusselt number was described with the aid of formula [10]

$$
\mathrm{Nu}_{i}=0.728\left(\frac{\rho_{l} g r_{i} D^{3}}{\mu_{l} \lambda_{l}\left(T_{m i}-T_{w}\right)}\right)^{0.25} .
$$

The ratio of coefficients of heat transfer for the condensation of water vapor with air and pure water vapor has been shown in Fig. 3 and described with the formula [2]

$$
\varepsilon=\frac{\alpha_{v a}}{\alpha_{v}}
$$

The dependence of $\varepsilon$ on the share of air for $k_{a} \in[0,0.07]$ was described with the 10th degree polynomial based on experimental data described in $[2]$

$$
\varepsilon=\sum_{j=0}^{10} a_{j} W_{j}\left(\varsigma_{i}\right),
$$

where $W_{j}$ is a Chebyshev polynomial $j[7]$, and the interval $k_{a} \in[0,0.07]$ was transformed to interval $\varsigma \in[-1,1]$. The values of the coefficients of 


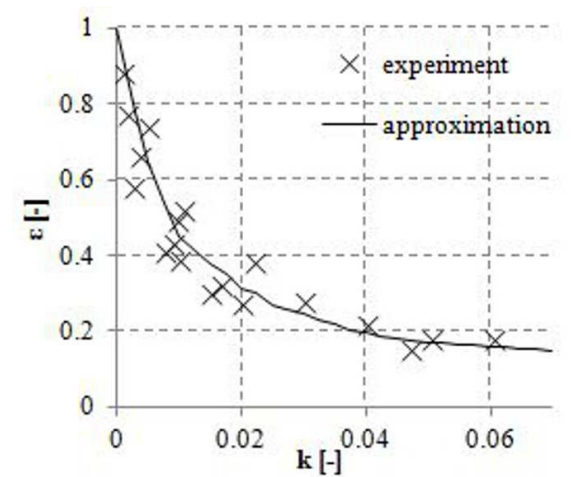

Figure 3: The dependence of $\varepsilon$ on the share of air [2].

Table 1: Polynomial coefficients.

\begin{tabular}{|l|l|l|r|l|l|}
\hline $\mathrm{a}_{0}$ & 0.354370 & $\mathrm{a}_{4}$ & 0.04383900 & $\mathrm{a}_{8}$ & -0.0019692 \\
\hline $\mathrm{a}_{1}$ & -0.319400 & $\mathrm{a}_{5}$ & -0.02284100 & $\mathrm{a}_{9}$ & 0.0025022 \\
\hline $\mathrm{a}_{2}$ & 0.172280 & $\mathrm{a}_{6}$ & 0.00787620 & $\mathrm{a}_{10}$ & -0.0016688 \\
\hline $\mathrm{a}_{3}$ & -0.085118 & $\mathrm{a}_{7}$ & -0.00029291 & & \\
\hline
\end{tabular}

the polynomial have been listed in Tab. 1. For the share of air above 0.07, a linear function was determined crossing the points $(0.07,0.15)$ and $(1,0)$. Based on the definition of Nusselt number and the value $\varepsilon$, the coefficient $\alpha$ was calculated allowing for determination of the share of air during condensation [2]

$$
\alpha_{v a i}=\varepsilon_{i} \frac{N u_{i} \lambda_{l}}{D} .
$$

The heat flow from the water vapor condensing to water inside is described by the formula

$$
Q_{i}=A_{p} \alpha_{v a i}\left(T_{m i}-T_{w}\right) .
$$

For the last row of tubes the heat flow was calculated based on the relation

$$
Q_{m}=r_{m-1}\left(\dot{m}_{m-1}-\dot{m}_{m}\right) \text {. }
$$

The flow of the condensed mass of water vapor is

$$
\Delta \dot{m}_{i}=\frac{Q_{i}}{r_{i}} .
$$




\section{Results of calculations}

The calculations were made with the assumption that the tube bank receives a mixture of water vapor and air of the pressure of $7000 \mathrm{~Pa}$ with the velocity of $17 \mathrm{~m} / \mathrm{s}$. Taking the change of the flow area in the tube bank into account, the velocity between the tubes in the first row was $34 \mathrm{~m} / \mathrm{s}$. Besides, it was assumed that there is air in the water vapor of the mass shares of: $0.002,0.004,0.006,0.008$, and 0.01 . The tube bank is composed of tubes set in a rectangular fashion of identical horizontal and vertical distances. The dimensions have been given in Tab. 2. The length of the tube bank results from the number of rows of tubes where the water vapor condenses.

Table 2: Geometry of the tube bank and input data for the calculations.

\begin{tabular}{|l|l|}
\hline$D, \mathrm{~m}$ & 0.028 \\
\hline$X, \mathrm{~m}$ & 0.056 \\
\hline$S, \mathrm{~m}$ & 1.008 \\
\hline$p_{m 0}, \mathrm{~Pa}$ & 7000 \\
\hline$c_{0}, \mathrm{~m} / \mathrm{s}$ & 17 \\
\hline$T_{w}, \mathrm{~K}$ & 283 \\
\hline$k,-$ & $0.002,0.004,0.006,0.008,0.01$ \\
\hline
\end{tabular}

The model allowed calculating the static pressure in the subsequent rows of tubes. For the increasing initial mass share of air from 0.002 to 0.01 the number of rows of tubes, on which total condensation of water vapor occurs, grows from 12 to 25 (Fig. 4a). The increase in the number of rows of tubes results in a greater loss of pressure during the flow of gas through the bank. The consequence of that is a drop of temperature (which depends on pressure) of water vapor saturation (Fig. 4b). This deteriorates the conditions of condensation.

When the mixture of gases flows through the tube bank, its velocity (Fig. 5a) and the Reynolds number (Fig. 5b) drops. This drop is the fastest for the lowest mass share of air upstream of the first row of tubes. In the last rows of the tube bank the water vapor fades. This results in a drop of the flow velocity of the mixture of gases and the Reynolds number to values close to zero. 


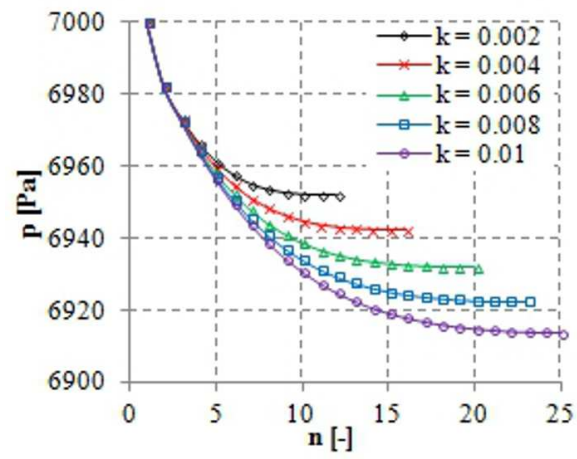

a)

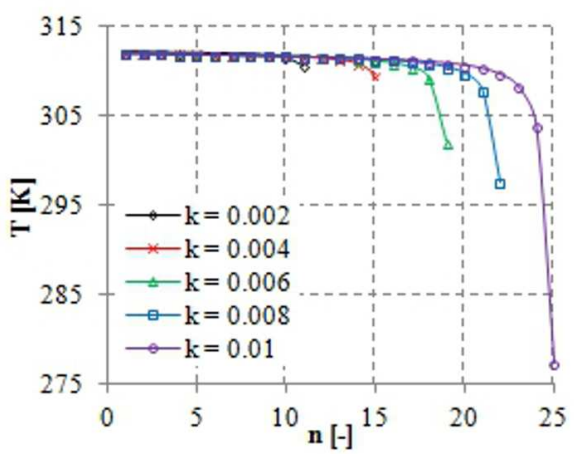

b)

Figure 4: Values of the pressure of the mixture of gases (a), and temperature of water vapor saturation in the subsequent rows of tubes (b) for the mass share of air upstream of the first row of tubes of $k=0.002,0.004,0.006,0.008$, and 0.01 .

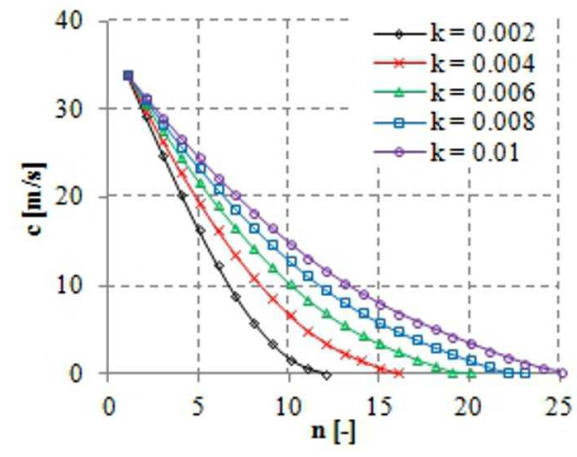

a)

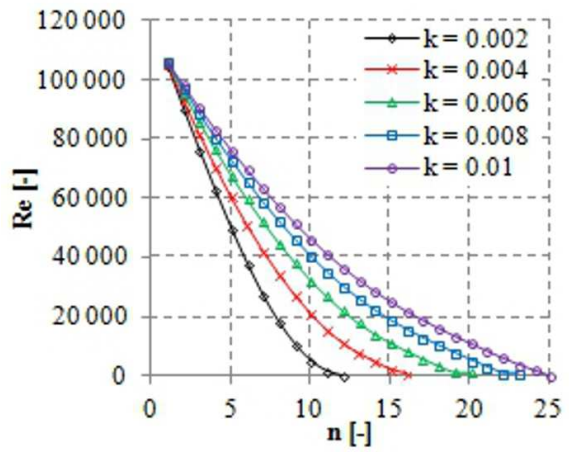

b)

Figure 5: Values of the velocities of gases (a), and Reynolds number in the subsequent rows of tubes (b) for the mass share of air upstream of the first row of tubes of $k=0.002,0.004,0.006,0.008$, and 0.01 .

The total loss coefficient, depending on the geometry of the tube bank and the Reynolds number, grows in subsequent rows of tubes to the value of approx. 30 (Fig. 6a). This growth is the fastest for the lowest initial share of air. A relatively high value of the total coefficient of pressure loss in the last rows of tubes results in a small loss of pressure due to low flow velocities of the mixture in these rows (Figs. 5a and 6). The drop of pressure is the greatest in the first row of tubes and it decreases in the subsequent rows of the tube bank (Fig. 6b). The total drop of pressure increases linearly along 
with the initial mass share of air (Fig. 7). For $k$ increasing from 0.002 to 0.01 the total static pressure loss grows from 48 to $85 \mathrm{~Pa}$.

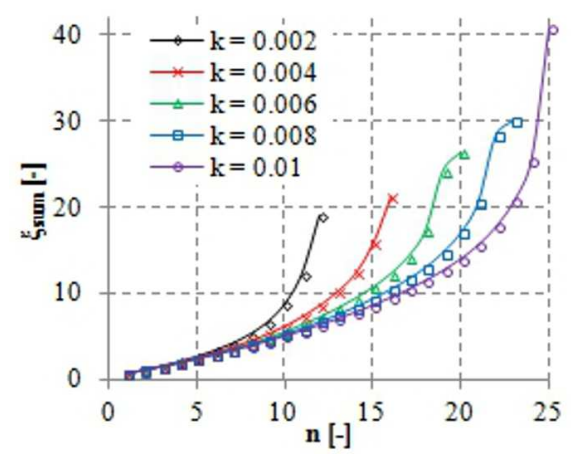

a)

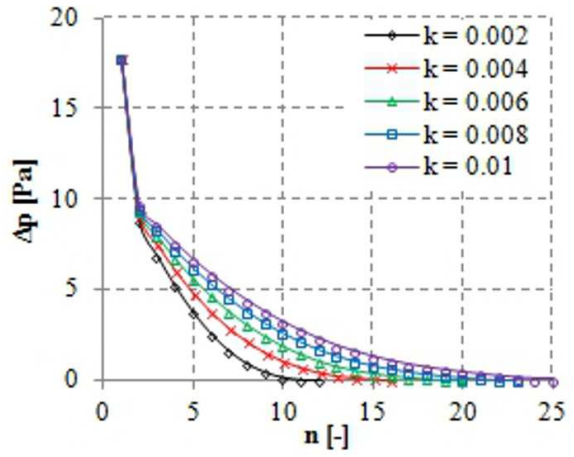

b)

Figure 6: Values of the total loss coefficient (a) and pressure loss in the subsequent rows of tubes (b) for the mass share of air upstream of the first row of tubes of $k=0.002,0.004,0.006,0.008$, and 0.01 .

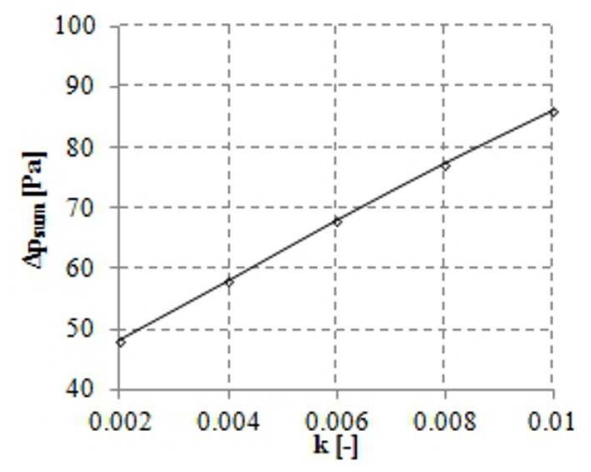

Figure 7: Values of the total drop of pressure of the mixture depending on the share of air upstream of the first row of tubes.

The partial pressure of water vapor, except the last rows, decreases smoothly in the model tube bank (Fig. 8a). The increment of partial pressure of air in this part of the tube bank has a similar character (Fig. 8b). In the last rows of tubes the partial pressure of water vapor drops abruptly and the partial pressure of air increases. This results from the small residual mass flow of water vapor and accumulation of air. 


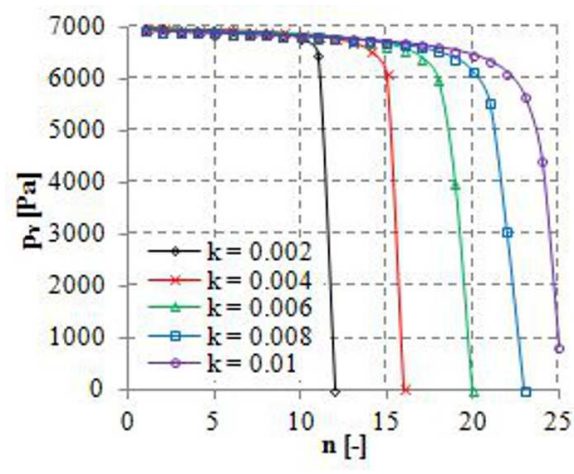

a)

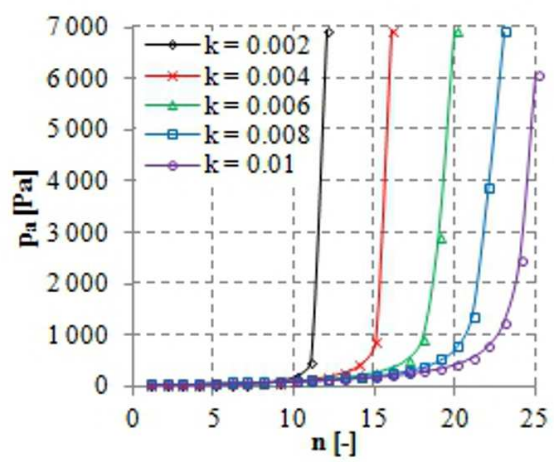

b)

Figure 8: Values of the partial pressure of water vapor (a) and partial pressure of air in the subsequent rows of tubes (b) for the mass share of air upstream of the first row of tubes of $k=0.002,0.004,0.006,0.008$, and 0.01 .

The heat of evaporation depends on the partial pressure of water vapor and the saturation temperature. For majority of rows, the heat of evaporation is approx. $2410 \mathrm{~kJ} / \mathrm{kg}$ (Fig. 9a). Due to a drop in the saturation, the value of the heat of evaporation in subsequent rows of tubes increases gradually. The Nusselt number for most of the rows assumes values of approx. 335 (Fig. 9b). In the last rows, an increase of the Nusselt number takes place, which results from a small difference between the temperature of the tube wall and the temperature of water vapor saturation. This increase also depends on the obtained course of heat of evaporation. The heat transfer coefficient $\alpha$ allowing for the presence of air in the condenser depends on the Nusselt number and the course of the function $\varepsilon$. The mass share of air upstream of the first row of tubes significantly influences the value of the heat transfer coefficient (Fig. 9c) and the heat flow (Fig. 9d) already at the front of the tube bank. For the first row of tubes, the heat transfer coefficient assumes the values from 3000 to $6000 \mathrm{~W} / \mathrm{m}^{2} \mathrm{~K}$ and the heat flow from 150 to $280 \mathrm{~kW}$. In the last rows of tubes the mass share of air $k$ grows rapidly as a result of the fading water vapor. This results in a decrease of heat transfer coefficient, $\alpha$.

The mass flow of gases flowing through the tube bank drops from approx. $0.8 \mathrm{~kg} / \mathrm{s}$ to values close to zero (Fig. 10a), which results from the condensation of water vapor. The amount of mass flow of condensed water vapor in the rows of the tube bank mainly depends on the heat transfer coefficient and the difference between the temperature of the wall and the 


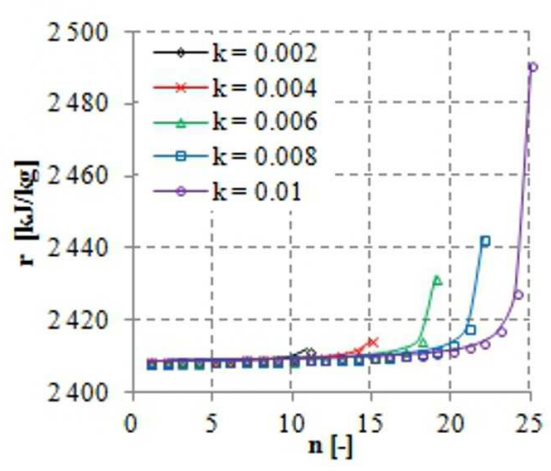

a)

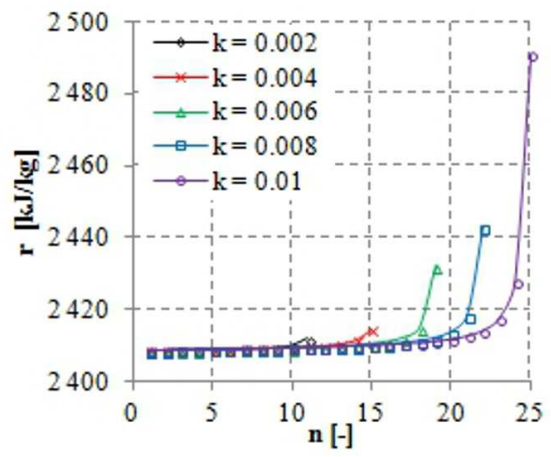

c)

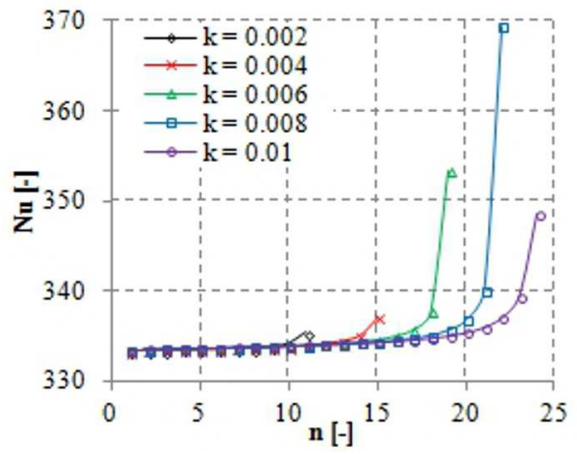

b)

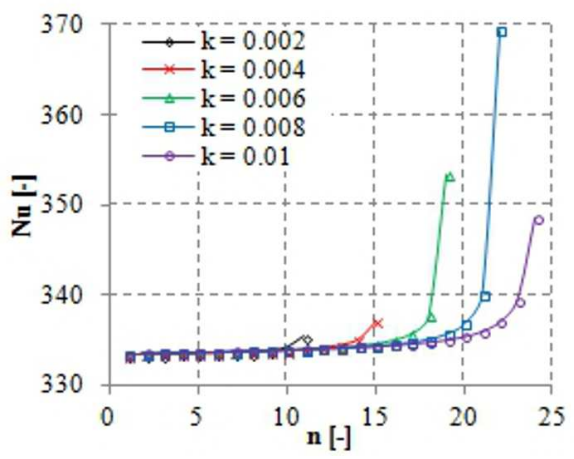

d)

Figure 9: Values of the heat of evaporation (a), Nusselt number (b), heat transfer coefficient (c), heat flow in the subsequent rows of tubes (d) for the mass share of air upstream of the first row of tubes of $k=0.002,0.004,0.006,0.008$, and 0.01 .

temperature of the mixture of gases. Hence, the courses of the characteristics of heat transfer coefficient, $\alpha$, and Nusselt number, Nu (Figs. 9c and 9d), and condensed water vapor mass flow rate, $\Delta \dot{m}$, (Fig. 10b) are similar. The mass flow rate of condensed water vapor decreases as the gases flow through the condenser (Fig. 10b). A deterioration of the conditions of condensation results from the increase of the heat resistance of the air accumulating in the subsequent rows of tubes (Fig. 11) and the drop of the partial pressure of water vapor, which results in a drop of the water vapor saturation temperature. In the last rows of tubes one can see an abrupt increase in the mass share of air, $k$, (Fig. 11). 


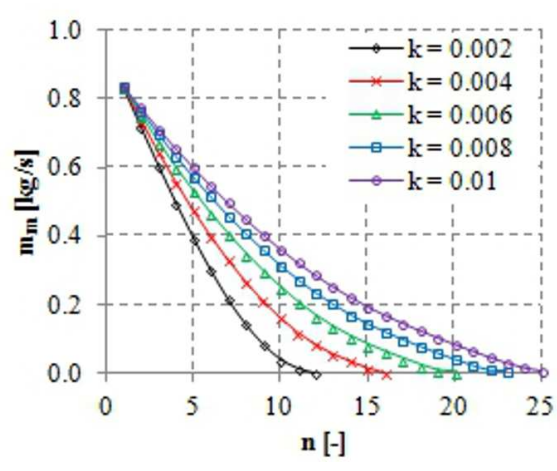

a)

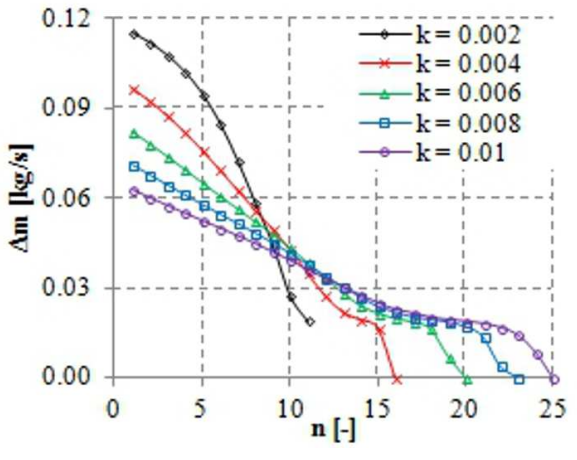

b)

Figure 10: Values of the mass flow rate of the mixture of gases (a), condensed water vapor mass flow in the subsequent rows of tubes (b) for the mass share of air upstream of the first row of tubes of $k=0.002,0.004,0.006,0.008$, and 0.01 .

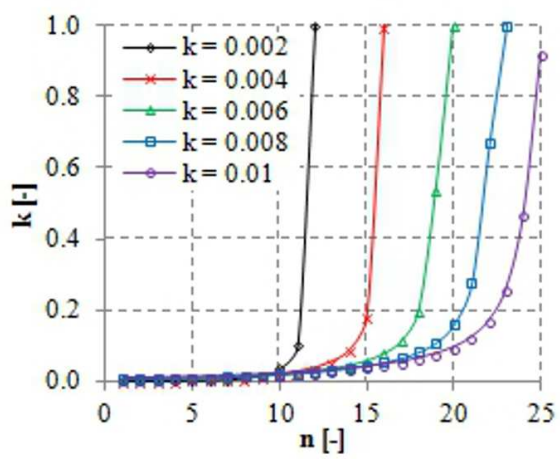

Figure 11: Values of the mass share of air in the subsequent rows of tubes for the mass share of air upstream of the first row of tubes of $k=0.002,0.004,0.006$, $0.008,0.01$

\section{Conclusions}

The presented calculation model allows determining of the average values of thermodynamic and flow parameters occurring in individual rows of tubes of a condenser. Based on the results included in the paper, it can be observed that a relatively small increase in the mass share of air in the water vapor entering the condenser results in a change of the flow parameters and a significant deterioration of the heat flow conditions. Additional heat resistance triggered by the accumulation of air results in a need to increase 
the number of rows of tubes for a successful condensation of water vapor to take place. The proposed calculation model allows determining of the length of the tube bank in which the water vapor condensation occurs.

The resultant length of the tube bank depends on the initial velocity, pressure of the mixture of gases and the mass share of air. The model also allows determining the row, in which the temperature of water vapor saturation is close to the temperature of the tube wall, in which case a significant deterioration or even pausing of the condensation process occurs. This and the knowledge of the mass share of air in each of the rows allow determining the spots where air accumulates in the tube bank of the condenser and its successful extraction.

The presented model assumes a constant value of the pressure and velocity in the intake plane to the model tube bank of the condenser. In the next stage of research, analysis is planned allowing for determination of the thermal and flow parameters in a tube bank of a condenser of a fixed number of rows and variable initial parameters of the air/water vapor mixture.

Received 8 August 2016

\section{References}

[1] Bin Ren, Li Zhang, Hong Xu, Jun Cao, Zhenyu Tao: Experimental study on condensation of steam/air mixture in a horizontal tube. Exp. Therm. Fluid Sci. 58(2014), 145-155.

[2] Hobler T.: Heat Transfer and Heat Exchangers. Warszawa, WNT 1986 (in Polish).

[3] Huiqiang Xu, Zhongning Sun, Haifeng Gu, Hao Li: Experimental study on the effect of wall-subcooling on condensation heat transfer in the presence of noncondensable gases in a horizontal tube. Ann. Nucl. Energy 90(2016), 9-21.

[4] JoAchimiak M., KrzyśLAK P.: The analysis of the changes in the partial pressure of gases and the share of air during a flow through a tube bank inside a condenser. JMTE 65(2013), 4, 13-21.

[5] Madejski J.: Heat Transfer Theory. PWN, Warszawa 1963 (in Polish).

[6] Marto P.J.: Heat Transfer and two-phase flow during shell-side condensation. Heat Transfer Eng. 5(1984), 1-2, 31-61.

[7] Paszkowski S.: Numerical Application of Multinomials and Chebyshev Series. PWN, Warszawa 1975 (in Polish).

[8] Rusowicz A.: Problems of Mathematical Modeling of Power Condensers. Oficyna Wydawnicza Politechniki Warszawskiej, Warszawa 2013 (in Polish).

[9] Wagner W., Kretzschmar H.J.: International Steam Tables, Properties of Water and Steam Based on the Industrial Formulation IAPWS-IF97. Springer-Verlag, 1998. 
[10] WiŚniewski S., WiŚniewski T.S.: Heat Transfer. WNT, Warszawa 2012 (in Polish).

[11] Release on the IAPWS Formulation 2008 for the Viscosity of Ordinary Water Substance. IAPWS, Berlin 2008. 